\title{
STRUCTURES OF CENTRAL BULGES AND \\ NUCLEI OF GALAXIES
}

\author{
G. DE VAUCOULEURS
}

Dept. of Astronomy, The University of Texas at Austin, Tex., U.S.A.

\begin{abstract}
The isophotal surfaces of central bulges of normal spirals, often described as 'spheroidal', are shown to depart from ellipsoids of revolution and to resemble MacLaurin or Jacobi spheroids with zonal distortion; two typical examples, NGC 4565, 5746, are illustrated. Weaker examples are NGC 2683, 4594 and 891. Stronger examples are NGC 7332 and 128.

The true nucleus of an ordinary spiral is at most a very small object $(\sim 10 \mathrm{pc})$ resolved only in the nearest galaxies. The apparently larger nuclei seen in more distant spirals and lenticulars, in particular the so-called $\mathrm{N}$-types, are probably artifacts due to insufficient resolution. It is possible that even in M31 the small 'nucleus' is merely the seeing-convolved image of the central peak in the $r^{1 / 4}$ luminosity distribution of the spheroidal component.

In the nearest barred lenticulars and early spirals each large ellipsoidal 'nucleus' $(\sim 1.0 \times 1.5 \mathrm{kpc})$ in the centre of the bar or central lens is found to include a second bar and inner nucleus on a smaller scale, in the approximate ratio of the corresponding Jeans' lengths. This is illustrated by high resolution photographs of NGC 1291 ; another example is NGC 1326.
\end{abstract}

\section{The Nuclear Bulge}

Among early-type spirals (Sa-Sb) a distinction should be made between systems with a dominant spheroidal component or large 'bulge', e.g. NGC 4594, and systems with a minimal nucleus and a small bulge, e.g. NGC 4866. This distinction has been noted in classification work (Sandage, 1961), but has no expression in the Hubble scheme in which it detracts from the usual close correlation between arm structure and bulge/ disk ratio. It is easily represented, however, by 2-parameter photometric models including spheroidal and exponential components.

A curious property of the nuclear bulge of spirals is the shape of the isophotal surfaces of the spheroidal component. These are usually described as ellipsoids of revolution. However, careful inspection of photographs of edge-on spirals and lenticulars discloses that the isophotes of the central bulge are often less convex near the minor axis than would be the case if they were true ellipses and are even slifghtly concave in a few cases. In other words they resemble MacLaurin or perhaps Jacobi spheroids with marked zonal distortions rather than simple ellipsoids of revolution. This is obvious in extreme cases such as NGC 128 whose isophotes are actually peanutshaped, and to a lesser extent in NGC 7332, both of which are lenticulars described as 'peculiar' with a 'box-shaped central region' in the Hubble Atlas (Sandage, 1961). A similar shape is also easily detectable in several edge-on spirals, such as NGC 891 and 5746 (Figure 1) and it is still perceptible although by no means obvious in many others, for example NGC $2683,4565,4594$. This shape may well be a general property of the spheroidal component which should be quantitatively accounted for by realistic dynamical models. 


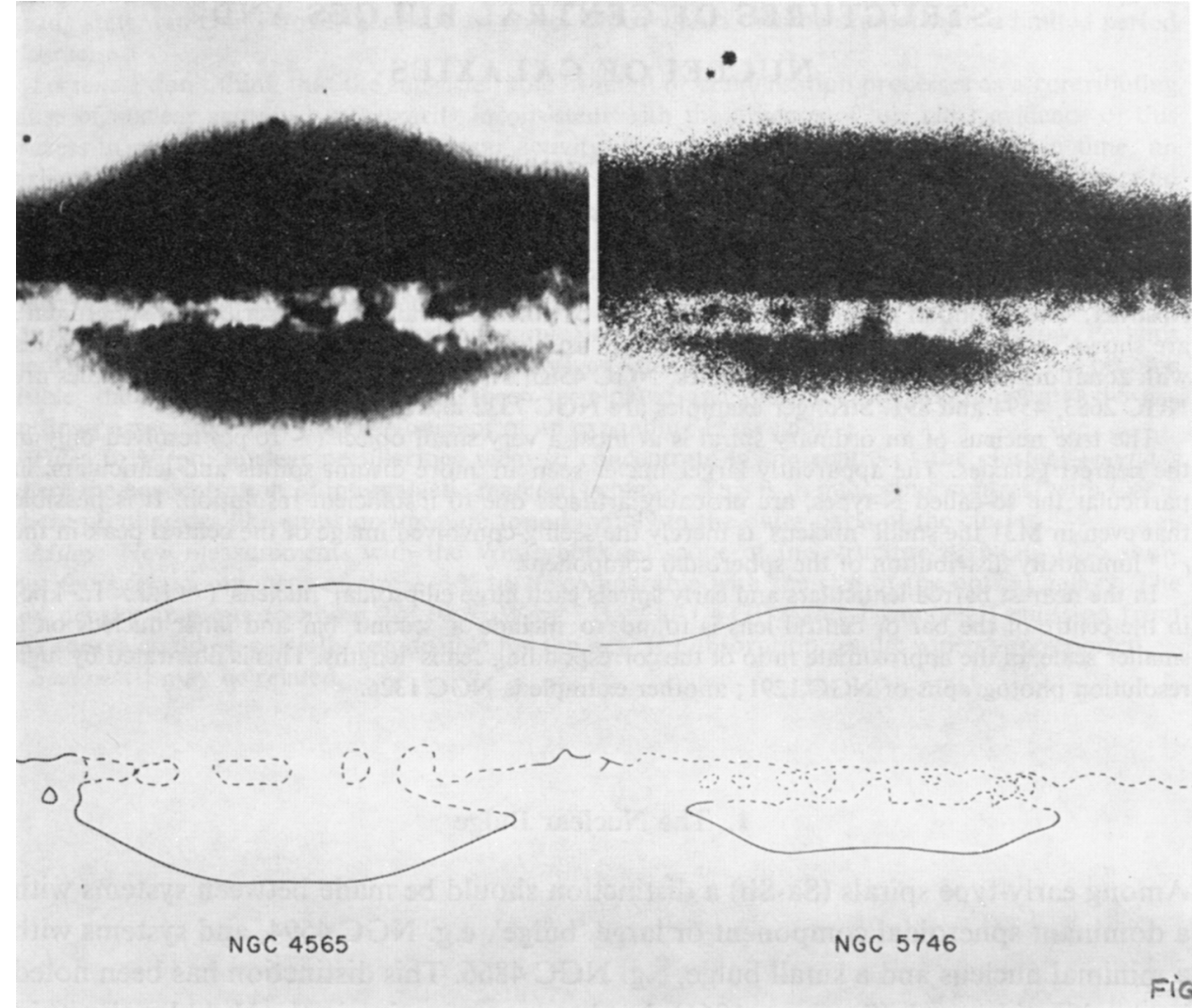

Fig. 1. The isophotes of the central bulges of NGC 4565 and NGC 5746 depart markedly from ellipses and resemble MacLaurin spheroids with zonal distortions.

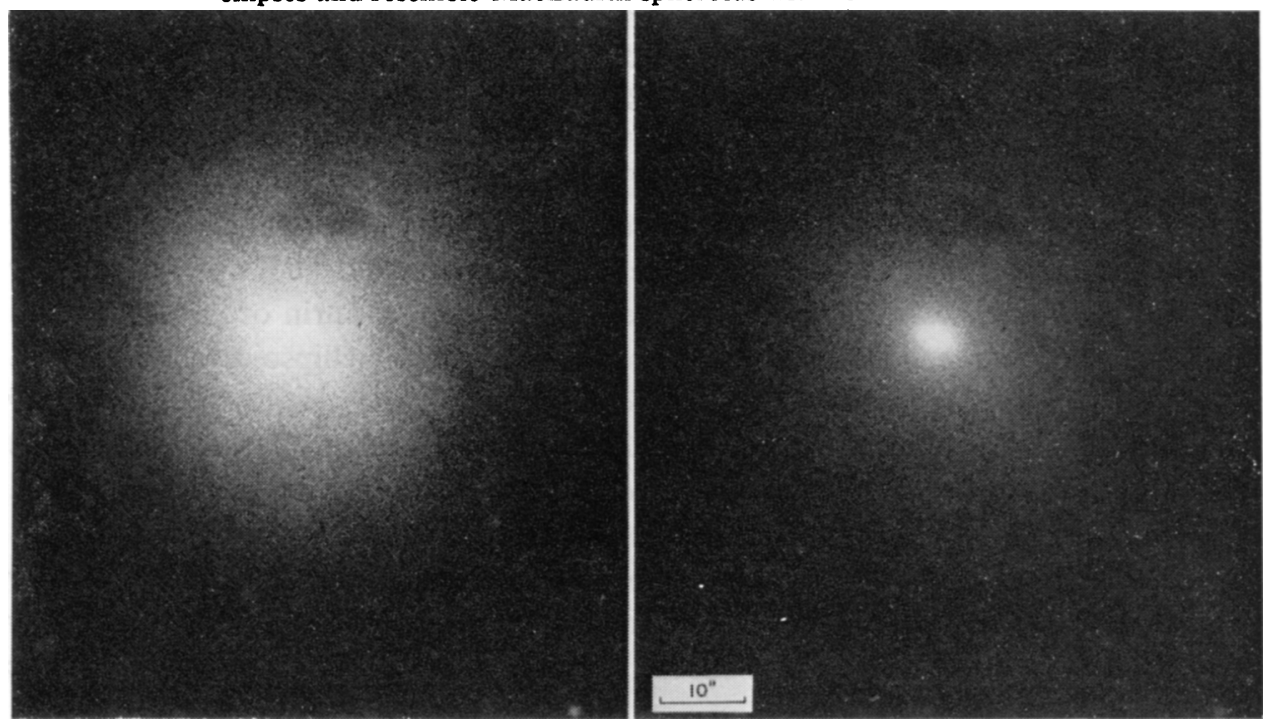

Fig. 2. The very small nucleus of M31 is a typical example of the central peak in the luminosity distribution of the spheroidal component of ordinary spirals. McDonald Observatory, $205-\mathrm{cm}$ Struve reflector, Cassegrain focus (1960 November 15, 103a-0, $10 \mathrm{~min}$; original scale: $7.15 \mathrm{~mm}^{-1}$ ). 


\section{Nuclei of Ordinary Lenticulars and Spirals}

In the nearest spirals (M31, M33) the high angular resolution available, relative to the size of the system, allows us to detect a very small ellipsoidal object (about 3". $3 \times 2$ ". $4=$ $=15 \times 10 \mathrm{pc}$ in M31; Figure 2) rather similar to a globular cluster in size although not in structure or composition (Johnson, 1961; Kinman, 1965).

If this object is a typical example of the true nuclei of ordinary spirals it would not be visible outside the Local Group; at the distance of M81 it would subtend less than $1^{\prime \prime}$, and less than 0.1 at the distance of the Virgo cluster. It is clear that the apparent

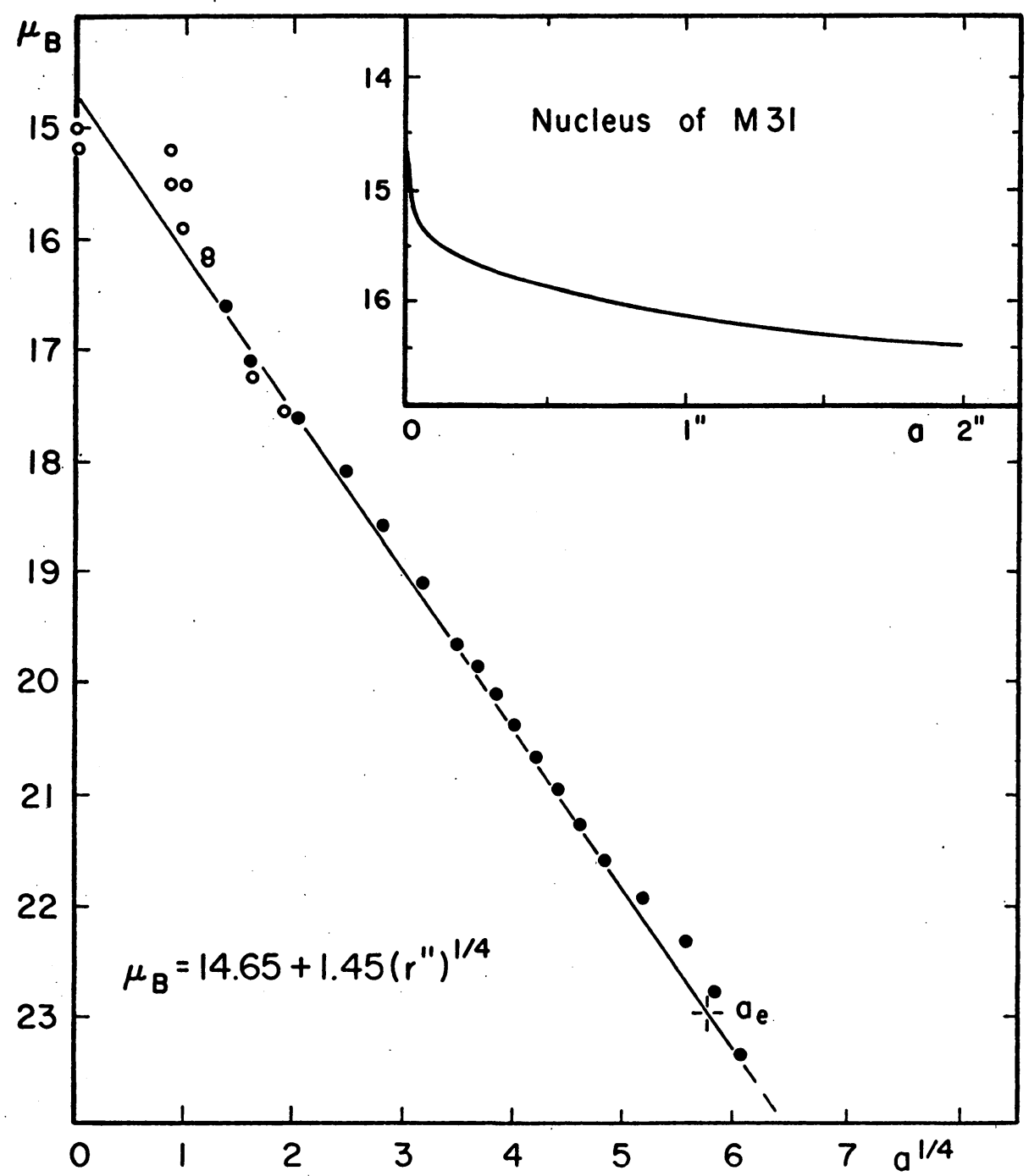

Fig. 3. The luminosity distribution of the spheroidal component of M31 along its major axis follows closely the characteristic $r^{1 / 4}$ law in the range $2^{\prime \prime}<a<20^{\prime}$ and, within the errors of roughly deconvolved photographic photometry, even into the 'nucleus'. 
'nucleus' seen on photographs of ordinary spirals and lenticulars is to a large extent an artifact due to insufficient resolution; in particular the appearance of many socalled $\mathrm{N}$-type galaxies arises from this effect, especially when a quasi-stellar central source is present. For example, high-resolution photoelectric scans of 3C 120 demonstrate that the luminosity profile of the bright 'nucleus' is not broader than the stellar seeing-disk profiles (de Vaucouleurs and de Vaucouleurs, 1968).

One may ask whether, even in M31, the small photographic nucleus (Figure 2) is really a distinct object and a separate photometric entity, or perhaps is merely the seeing-convolved image of the central peak in the very steep luminosity distribution of the bulge, which is known to follow closely the $r^{1 / 4}$ law of the spheroidal component at least to $a \simeq 20^{\prime}$ along the major axis (de Vaucouleurs, 1958).

A plot (Figure 3) of available photoelectric and corrected photographic data (Johnson, 1951; Kinman, 1965) shows that, except perhaps within 2" from the centre where both the original photometry and the (approximate) deconvolution corrections are uncertain, the luminosity distribution follows closely the relation

$$
\mu_{B}=14.65+1.45 r^{1 / 4} \text {, }
$$

where $\mu_{B}$ is the $B$-system mag (arc sec) $)^{-2}$ and $r$ is in arc sec. The corresponding effective radius is $a_{\mathrm{e}}=(3.33 \times 2.5 / 1.45)^{4}=18^{\prime}$ where $\mu_{B}=\mu(0)+8.32=22.97 \mathrm{mag}(\operatorname{arcsec})^{-2}$. If the relation holds right up to the centre, the central 1.0 mag spike from $\mu_{B} \simeq 15.65$ at $a \simeq 0$ ". 2 to $\mu_{B} \simeq 14.65$ at $a=0$ (Figure 3, inset) might be detectable on the Stratoscope II photographs.

\section{Nuclei of Barred Lenticulars and Spirals}

In barred spirals the situation is entirely different; here the nucleus is generally a fairly large, distinct ellipsoidal object with typical dimensions of the order of $1.5 \times 1.0 \mathrm{kpc}$, comparable to the half-width of the bar. This appearance is confirmed by detailed surface photometry, e.g. in NGC 1291, 1433, 1512, etc., which indicates a fairly sharp change of slope in the luminosity profile at the edge of the nucleus. The laws of luminosity distribution in these nuclei are not yet precisely known, because few barred spirals are near enough for high-resolution quantitative analysis. Nevertheless, a remarkable structure, not previously reported, has been noted on high-resolution photographs of the nuclei of two of the largest, nearby, barred systems, NGC 1291 and 1326, which are both classified as (R) SB(s) 0/a, i.e. transition types between late lenticulars and early barred spirals with conspicuous outer rings. Inside the elliptical nucleus at the centre of the bar crossing the primary lens, a second, smaller-scale bar structure is present along the major axis of the large nucleus, or secondary lens, in the centre of which is a second, much smaller nucleus. In other words the lens-bar-nucleus pattern is repeated on two different scales, one inside the other (Figure 4). This repetitive pattern suggests that the nucleus is so much denser than the main bar and lens that its dynamics is almost independent of the extra-nuclear mass distribution, and that the same mechanisms are at work to produce similar structures with different 

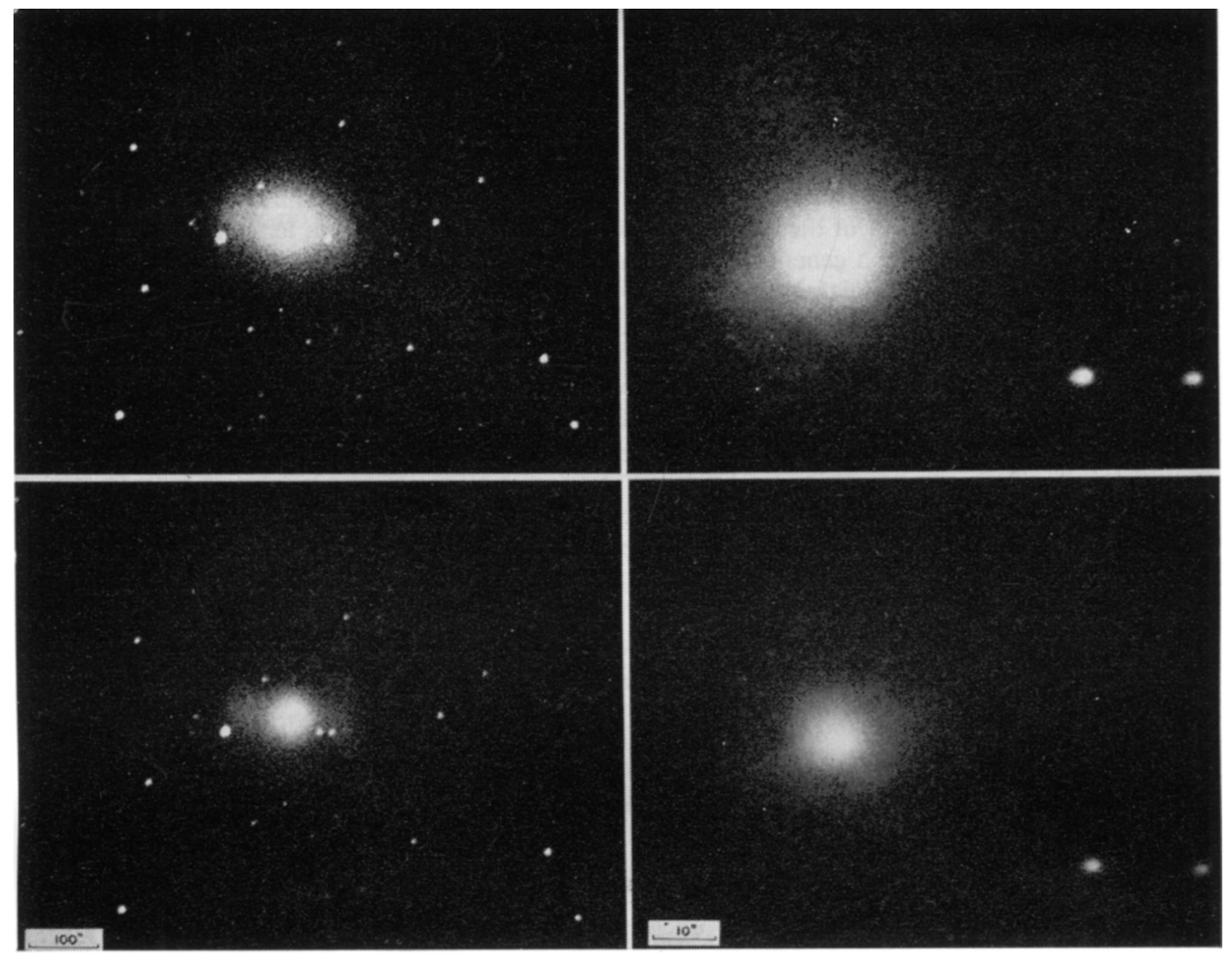

Fig. 4. The lens-bar-nucleus pattern is repeated on two different scales in the large southern barred galaxy NGC 1291, revised type (R) SB(s) 0/a.

At left: the primary lens, bar and 'nucleus' are visible on this small scale photograph. Newtonian focus of Mt. Stromlo Observatory 75-cm Reynolds reflector (1952 November 19, 103a-0, 60 min; original scale: $67^{\prime \prime} \mathrm{mm}^{-1}$ ).

At right: the secondary lens, bar and inner nucleus are shown on this large scale photograph of the internal structure of the primary 'nucleus'. Cassegrain focus of McDonald Observatory 205-cm Struve reflector (1960 November 14, 103a-0, $65 \mathrm{~min}$; original scale: 7 ".5 $\mathrm{mm}^{-1}$ ). Elongated star images are caused by atmospheric dispersion at $z \simeq 73^{\circ}$.

scale factors measured, e.g. by the Jeans' length $L=\left(\sigma_{\mathrm{v}}^{2} / G \varrho\right)^{1 / 2}$. If $\sigma_{\mathrm{v}}=$ const., $L \propto \varrho^{-1 / 2}$. Since the mean surface brightness, and presumably the mean density $\varrho$, in the nucleus of NGC 1291 is about 40 times higher than in the lens, the Jeans' length is 6 to 7 times smaller in the nucleus than in the lens, in approximate agreement with the scale ratio of the two bar structures.

\section{References}

de Vaucouleurs, G.: 1958, Astrophys. J. 128, 465.

de Vaucouleurs, G. and de Vaucouleurs, A.: 1968, Astron. J. 73, S174.

Johnson, H. M.: 1961, Astrophys. J. 133, 309.

Kinman, T. D.: 1965, Astrophys. J. 142, 1376.

Sandage, A.: 1961, The Hubble Atlas of Galaxies, Carnegie Inst., Washington, D. C. 


\section{DISCUSSION}

Carrick: I am working on this very subject, the light distributions of the bulges of edge-on systems. Of the six disk galaxies for which I have plates, two definitely show this box-like shape. It appears to be a general phenomenon.

G. de Vaucouleurs: Yes, you can see it plainly on photographs of edge-on spirals such as NGC 891, and Sandage has pointed out in the Hubble Atlas a similar phenomenon in lenticulars, particularly in NGC 7332. So it must be a general phenomenon and theoreticians should explain it.

Mark: There is a possible analogy here with rotating stars. If stars are rotating differentially then one can get isophotes which are 'box-like', though not if they are rotating uniformly. It is possible that, by using the moment equations for stellar dynamics instead of the fluid equations of stellar structure, differential rotation in galactic bulges could give rise to box-like isophotes.

Butcher: May I ask Dr de Vaucouleurs to comment on some observations by Zasov in Moscow, which suggest that the luminosity gradients of the more extreme Seyfert galaxies are steeper than in normal spirals of the same types.

G. de Vaucouleurs: I have not seen this paper, but we have recently completed a detailed study of all classical Seyferts which does not seem to support this view. When the luminosity of the nuclear point source is correctly subtracted, Seyfert galaxies appear normal for their morphological types with respect to luminosity gradients and colours; in one or two cases only is there perhaps a very slight negative colour excess in the innermost regions. This is difficult to assess exactly because of the variable amounts of line emission in the disk. 